\title{
Simultaneous Robust Fault and State Estimation for Linear Discrete-Time Uncertain Systems
}

\author{
Feten Gannouni and Fayçal Ben Hmida \\ National Higher School of Engineering of Tunis (ENSIT), Laboratoire d'Ingenierie des Systèmes Industriels et \\ des Energies Renouvelables (LISIER), University of Tunis, 5 Taha Hussein Street, BP 56, 1008 Tunis, Tunisia
}

Correspondence should be addressed to Feten Gannouni; fetengannouni@yahoo.fr

Received 30 July 2016; Revised 8 October 2016; Accepted 24 October 2016; Published 15 January 2017

Academic Editor: Alberto Borboni

Copyright ( 2017 F. Gannouni and F. Ben Hmida. This is an open access article distributed under the Creative Commons Attribution License, which permits unrestricted use, distribution, and reproduction in any medium, provided the original work is properly cited.

\begin{abstract}
We consider the problem of robust simultaneous fault and state estimation for linear uncertain discrete-time systems with unknown faults which affect both the state and the observation matrices. Using transformation of the original system, a new robust proportional integral filter (RPIF) having an error variance with an optimized guaranteed upper bound for any allowed uncertainty is proposed to improve robust estimation of unknown time-varying faults and to improve robustness against uncertainties. In this study, the minimization problem of the upper bound of the estimation error variance is formulated as a convex optimization problem subject to linear matrix inequalities (LMI) for all admissible uncertainties. The proportional and the integral gains are optimally chosen by solving the convex optimization problem. Simulation results are given in order to illustrate the performance of the proposed filter, in particular to solve the problem of joint fault and state estimation.
\end{abstract}

\section{Introduction}

This paper is concerned with the problem of joint fault and state estimation of linear discrete-time uncertain systems under convex bounded parametric uncertainty. This problem is solved by using a robust filtering approach to produce a robust fault and state estimation [1-6].

The proposed filter can play a significant role in several applications, for example, model based fault detection and isolation (FDI) problem [1-7] and fault tolerant control (FTC) problem $[7,8]$.

In the past three decades, the problem of robust state estimation in the presence of uncertainties has attracted the interests of many researchers. This problem is largely treated in the literature by different approaches: the guaranteed cost design [9-12], the $H_{\infty}$ filtering [13-15], and the set-valued estimation $[16,17]$. One limitation of the different design approach for online filter operation is that they require continuous testing of a certain existence condition. When the condition fails at any particular iteration, the proposed filters can diverge.
From the point of view of minimizing the worst possible regularized residual norm over the class of admissible uncertainties, new robust filters are designed for linear uncertain systems by $[18,19]$. Compared with the aforementioned robust formulations the developed filters perform data regularization rather than deregularization which represent an important property for online operation. The proposed filters in [19] are based in data regularization solution. This filter guarantees an error variance with an optimized guaranteed upper bound for any allowed uncertainty. To improve robustness against uncertainties such as disturbances and modeling errors, [20] introduced a proportional integral Kalman filter (PIKF). The proportional and the integral Kalman gains were obtained from the solution of Riccati equation leading to minimum error variance. Later, [21] developed a new robust proportional integral Kalman filter for stochastic linear uncertain systems. The filtering problem is converted into a convex optimization problem for continuous time systems with polytopic uncertainties and the filter parameters are optimally chosen by solving this problem. 
The problem of robust Kalman filtering and optimal filtering in the presence of unknown inputs and unknown faults has received considerable attention in the last two decades due to its significations role in many applications, for example, geophysical and environmental applications, fault detection and isolation (FDI) problems, and fault tolerant control (FTC) problems.

The FDI (fault detection and isolation) problem for linear systems with unknown disturbances is largely studied in the literature by different approaches; see, for example, [2-7] and $[22,23]$. By using the error innovation technique, a robust fault detection and isolation filter in continuous time is developed in [22] to generate unbiased white residuals signals. In [23] a new method is developed for linear time-invariant (LTI) stochastic discrete-time systems with unknown inputs. This method is important to detect and isolate multiple faults appearing simultaneously or sequentially in linear timeinvariant (LTI) systems.

The optimal filtering and robust fault diagnosis problem has been treated for stochastic systems with unknown disturbances in $[6,7]$. An optimal observer is proposed for linear time-varying systems. This observer can produce disturbances decoupled state estimation with minimum-variance. The output estimation error with disturbance decoupling $[6,7]$ is used as a residual signal. After that, a statistical testing procedure is applied to examine the residual and to diagnose faults. Nevertheless, the simultaneous actuator and sensor faults problem is not considered in $[6,7]$.

More recently, [3] presents a new recursive filter to joint fault and state estimation of linear time-varying discretetime systems in the presence of unknown disturbances. The method is based on the assumption that no prior knowledge about the dynamical evolution of the fault and the unknown disturbances is available. The filter considers an arbitrary direct feedthrough matrix of the fault and it permits a multiple faults estimations. However, the obtained filter may in certain cases suffer from poor quality fault estimation.

Later, in [24] the problem of joint fault and state estimation of linear systems in the presence of unknown input with uncertain noise covariances was presented. This problem was solved by using the proportional integral three-stage Kalman filter (PI-ThSKF) to estimate the state and the fault of stochastic discrete-time systems with unknown inputs. However, this approach assumes that the models for the dynamical evolution of the fault and the unknown inputs are available.

Based on the assumption that no prior knowledge about the dynamical evolution of the fault is available, the same author [25] was proposed a new recursive optimal filter structure with transformation of the original system. A new recursive optimal unbiased minimum-variance filter has been developed when the direct feedthrough matrix of the fault has an arbitrary rank. However, the filtering algorithm requires the knowledge of a perfect dynamic model. Thus the developed filter may not be robust against modeling uncertainty in the state and the output matrices.

One limitation of the proposed design approach $[3,25]$ is that they require testing of a certain existence conditions. When the condition fails at any particular iteration, the desired performance is lost and the filter can diverge. In addition the disadvantages of the existing approaches [3, 4, $24,25]$ are that the filter lost its optimality in the presence of uncertainties in the state and the output matrices.

In this paper, we consider the problem of robust joint fault and state estimation for linear discrete-time systems with norm bounded uncertainties in both the state and output matrices. The problem addressed is the design of robust linear filters that bound the state covariance matrix for all admissible uncertainties. It is shown that a robust proportional filter (RPF) is developed using transformation of the original system. This transformation is based on the singular value decomposition of the direct feedthrough matrix distribution of the fault which assumed to be arbitrary rank. The proposed filter guarantees that the variance of the estimation error is not more than an optimized upper bound for all admissible uncertainties. The minimization problem of the upper bound on the estimation error variance is formulated as a convex optimization problem subject to linear matrix inequalities and the filter parameters are optimally chosen by solving this problem. To improve robustness against uncertainties and to improve robust estimation of unknown time-varying fault, a new robust proportional integral filter (RPIF) is proposed. The proportional and the integral gains are optimally chosen by solving a convex optimization problem. So the resulted filter will be applied to solve a simultaneous actuator and sensor faults estimation problem.

The remainder of this paper is organized as follows. In Section 2 we set up the robust regularized least square problem for models with data uncertainties. Section 3 states the problem of interest. In Section 4 we design the robust proportional filter. Next in Section 5 we propose a design approach for the robust proportional integral filter (RPIF). Finally, in Section 6, the estimation performance of the proposed filters is demonstrated through an illustrative example.

\section{Preliminaries}

Consider the following optimization problem:

$$
\min _{x} \max _{\{\delta A, \delta b\}}\left[\|x\|_{\Pi}^{2}+\|(A+\delta A) x-(b+\delta b)\|_{W}^{2}\right]
$$

where $A$ is the data matrix, $b$ is the measurement vector which is assumed to be known, $x$ is the unknown vector, $\Pi=$ $\Pi^{T} \geq 0$ and $W=W^{T}>0$ are given weighting matrices, and $\{\delta A, \delta b\}$ are uncertainties assumed to satisfy a model of the form:

$$
\left[\begin{array}{ll}
\delta A & \delta b
\end{array}\right]=H \Delta\left[\begin{array}{ll}
N_{a} & N_{b}
\end{array}\right]
$$

where $\Delta$ is an arbitrary contraction, $\|\Delta\| \leq 1$, and $\left\{H, N_{a}\right.$, $\left.N_{b}\right\}$ are known quantities of appropriate dimension.

Problem (1) and (2) has a unique solution $\widehat{x}_{k}$ that is given by [17]:

$$
\widehat{x}=\left[\widehat{\Pi}+A^{T} \widehat{W} A\right]^{-1}\left[A^{T} \widehat{W} b+\widehat{\lambda} N_{a}^{T} N_{b}\right]
$$


where the modified weighting matrices $\{\widehat{\Pi}, \widehat{W}\}$ are defined by

$$
\begin{aligned}
& \widehat{\Pi}=\Pi+\widehat{\lambda} N_{a}^{T} N_{a}, \\
& \widehat{W}=W+W H\left(\widehat{\lambda} I-H^{T} W H\right)^{\dagger} H^{T} W
\end{aligned}
$$

and $\hat{\lambda}$ is a nonnegative scalar parameter obtained by the following optimization problem:

$$
\hat{\lambda}=\arg \min _{\hat{\lambda} \geq\left\|H^{T} W H\right\|} G(\lambda),
$$

where

$$
\begin{aligned}
G(\lambda):= & \|x(\lambda)\|_{\Pi}^{2}+\lambda\left\|N_{a} x(\lambda)-N_{b}\right\|^{2} \\
& +\|A x(\lambda)-b\|_{W(\lambda)}^{2} .
\end{aligned}
$$

\section{Problem Statement}

Consider the linear stochastic uncertain discrete-time system with unknown additive fault in the form:

$$
\begin{aligned}
x_{k+1} & =\left(A_{k}+\Delta A_{k}\right) x_{k}+F_{k}^{x} f_{k}+B_{k} u_{k}+w_{k}, \\
y_{k} & =\left(C_{k}+\Delta C_{k}\right) x_{k}+F_{k}^{y} f_{k}+v_{k},
\end{aligned}
$$

where $x_{k} \in \mathfrak{R}^{n}$ is the state vector, $y_{k} \in \mathfrak{R}^{p}$ is the observation vector, $f_{k} \in \mathfrak{R}^{m}$ is the unknown additive fault vector, $w_{k}$ and $v_{k}$ are uncorrelated white noise sequences of zero-mean and with covariances matrices $Q_{k}=E\left[w_{k} w_{l}^{T}\right] \geq 0$ and $R_{k}=$ $E\left[v_{k} v_{l}^{T}\right]>0$, respectively.

The matrices $A_{k}, B_{k}, C_{k}, F_{k}^{x}$, and $F_{k}^{y}$ are known and have appropriate dimensions.

The initial state $x_{0}$ is a Gaussian random variable that is uncorrelated with $\left\{w_{k}, v_{k}\right\}$ for all $k$ with $E\left[x_{0}\right]=\hat{x}_{0}$ and $E\left[\left(x_{0}-\hat{x}_{0}\right)\left(x_{0}-\hat{x}_{0}\right)^{T}\right]=P_{0}^{x}$, where $E[\cdot]$ denote the expectation operator.

$\Delta A_{k}$ and $\Delta C_{k}$ are unknown matrices which represent time-varying parameter uncertainties. These uncertainties are assumed to be of the following structure:

$$
\left[\begin{array}{c}
\Delta A_{k} \\
\Delta C_{k}
\end{array}\right]=\left[\begin{array}{c}
H_{1, k} \\
H_{2, k}
\end{array}\right] F_{k} E_{k}
$$

where $H_{1, k}, H_{2, k}$, and $E_{k}$ are known time-varying matrices of appropriate dimensions, while $F_{k}$ is an unknown timevarying matrix satisfying arbitrary contraction, $F_{k} F_{k}^{T} \leq$ $I, \forall k \in\left[\begin{array}{ll}0 & N\end{array}\right]$.

The aim of this paper is to design a new robust proportional integral filter (RPIF) to obtain a robust fault and state estimation when $0<\operatorname{rank}\left[F_{k}^{y}\right] \leq m$ in spite of the presence of parametric uncertainties.

Initially, we seek to change the coordinate of system (7) by using the same technique developed in [26].

Let $r_{k}=\operatorname{rank}\left(F_{k}^{y}\right)<m$, and then the singular value decomposition of the matrix $F_{k}^{y}$ is given by

$$
F_{k}^{y}=\left[\begin{array}{ll}
U_{1, k} & U_{2, k}
\end{array}\right]\left[\begin{array}{cc}
\Sigma_{k} & 0 \\
0 & 0
\end{array}\right]\left[\begin{array}{c}
V_{1, k}^{T} \\
V_{2, k}^{T}
\end{array}\right],
$$

where $\Sigma_{k} \in \mathfrak{R}^{r_{k} \times r_{k}}, U_{1, k} \in \mathfrak{R}^{p \times r_{k}}, U_{2, k} \in \mathfrak{R}^{p \times\left(p-r_{k}\right)}, V_{1, k} \in$ $\mathfrak{R}^{m \times r_{k}}$, and $V_{2, k} \in \mathfrak{R}^{m \times\left(m-r_{k}\right)}$. [ $\left[\begin{array}{ll}U_{1, k} & U_{2, k}\end{array}\right]$ and $\left[\begin{array}{ll}V_{1, k} & V_{2, k}\end{array}\right]$ are unitary matrices.

Using the notations

$$
\begin{aligned}
f_{k} & =V_{1, k} f_{1, k}+V_{2, k} \bar{f}_{2, k}, \\
F_{1, k}^{x} & =F_{k}^{x} V_{1, k}, \\
\bar{F}_{2, k}^{x} & =F_{k}^{x} V_{2, k}, \\
F_{1, k}^{y} & =U_{1, k} \Sigma_{k},
\end{aligned}
$$

where $f_{1, k}=V_{1, k}^{T} f_{k}$ and $\bar{f}_{2, k}=V_{2, k}^{T} f_{k}$, we obtain the following equivalent system of the original system (7).

$$
\begin{aligned}
x_{k+1}= & \left(A_{k}+\Delta A_{k}\right) x_{k}+F_{1, k}^{x} f_{1, k}+\bar{F}_{2, k}^{x} \bar{f}_{2, k}+B_{k} u_{k} \\
& +w_{k}, \\
y_{k}= & \left(C_{k}+\Delta C_{k}\right) x_{k}+F_{1, k}^{y} f_{1, k}+v_{k},
\end{aligned}
$$

where $F_{1, k}^{y}$ is of full-column rank due to (11).

Note that $\bar{F}_{2, k}^{x}$ in (12) may not have full-column rank and the unknown fault $\bar{f}_{2, k}$ may not be estimable. However it can be solved by finding a full-rank factorization of $\bar{F}_{2, k}^{x}$, that is, $\bar{F}_{2, k}^{x}=F_{2, k} \breve{F}_{2, k}$, where $F_{2, k}$ is $n \times r$ full-column rank matrix and $\breve{F}_{2, k}$ is $r\left(m-r_{k}\right)$ full-row rank matrix [27].

Thus by defining the notation

$$
f_{2, k}=\breve{F}_{2, k}^{x} \bar{f}_{2, k}
$$

(12) becomes

$$
\begin{aligned}
x_{k+1}= & \left(A_{k}+\Delta A_{k}\right) x_{k}+F_{1, k}^{x} f_{1, k}+F_{2, k}^{x} f_{2, k}+B_{k} u_{k} \\
& +w_{k} .
\end{aligned}
$$

\section{Robust Proportional Filter Design}

In this section, we propose to solve equivalent system (13), (15) for $\hat{x}_{k / k}, \hat{f}_{1, k / k}$ and $\hat{f}_{2, k-1 / k}$, such that $\operatorname{trace}\left\{E\left[e_{k} e_{k}^{T}\right]\right\}$ is minimized, where $e_{k}=x_{k}-\hat{x}_{k / k}$ is the state estimation error.

Here, we adopt a robust least-squares estimation approach to obtain a robust estimate for the state variable and the unknown faults by following a two-step procedure.

4.1. Bounded Uncertainties in $C_{k}$ Alone. Assume first that there are no uncertainties in $A_{k}$; we will incorporate the uncertainties in $A_{k}$ later. With bounded uncertainties in the output matrix $C_{k}$ alone and by using the robust least-squares estimation procedure, we can transform the original system 
(13), (15) into the following augmented output equation (AOE):

$$
\begin{aligned}
& {\left[\begin{array}{c}
y_{k}-\left(C_{k}+\Delta C_{k}\right) \hat{x}_{k / k-1} \\
0
\end{array}\right]} \\
& =\left[\begin{array}{ccc}
\left(C_{k}+\Delta C_{k}\right) & F_{1, k}^{y} & 0 \\
I & 0 & -F_{2, k-1}^{x}
\end{array}\right]\left[\begin{array}{c}
x_{k}-\widehat{x}_{k / k-1} \\
f_{1, k / k} \\
f_{2, k-1 / k}
\end{array}\right] \\
& +\left[\begin{array}{c}
v_{k} \\
-w_{k-1}
\end{array}\right]+\left[\begin{array}{cc}
0 & 0 \\
A_{k-1, c} & F_{1, k-1}^{x}
\end{array}\right] v_{k-1},
\end{aligned}
$$

where

$$
\begin{aligned}
\hat{x}_{k / k-1} & =A_{k-1} \hat{x}_{k-1 / k-1}+B_{k-1} u_{k-1}+F_{1, k-1}^{x} \hat{f}_{1, k-1 / k-1}, \\
v_{k} & =-\left[\begin{array}{ll}
e_{k}^{T} & \tilde{f}_{1, k}^{T}
\end{array}\right]^{T},
\end{aligned}
$$

where $\tilde{f}_{1, k}=f_{1, k}-\hat{f}_{1, k / k}$. Here $\hat{f}_{1, k / k}$ is the estimate of the fault and $v_{k}$ is zero-mean Gaussian and independent of $w_{k}$ and $v_{k+1}$.

The filtered estimate $\hat{x}_{k / k}$, the unknown fault $\hat{f}_{1, k / k}$, and the delayed unknown fault $\widehat{f}_{2, k-1 / k}$ can be obtained by solving the following robust least-squares problem:

$$
\min _{x_{k}, f_{1, k}, f_{2, k-1}} \max _{\Delta C_{k}}\left\|\left[\begin{array}{c}
y_{k}-\left(C_{k}+\Delta C_{k}\right) \hat{x}_{k / k-1} \\
0
\end{array}\right]-\left[\begin{array}{ccc}
\left(C_{k}+\Delta C_{k}\right) & F_{1, k}^{y} & 0 \\
I & 0 & -F_{2, k-1}^{x}
\end{array}\right]\left[\begin{array}{c}
x_{k}-\widehat{x}_{k / k-1} \\
f_{1, k / k} \\
f_{2, k-1 / k}
\end{array}\right]\right\|_{\operatorname{diag}\left(R_{k}^{-1}, \bar{P}_{k}^{-1}\right)},
$$

where

$$
\begin{aligned}
\bar{P}_{k} & =E\left[\left(x_{k}-\widehat{x}_{k / k-1}\right)\left(x_{k}-\widehat{x}_{k / k-1}\right)^{T}\right] \\
& =\left[\begin{array}{ll}
A_{k-1} & F_{1, k-1}^{x}
\end{array}\right]\left[\begin{array}{ll}
P_{k-1} & P_{k-1}^{x f_{1}} \\
P_{k-1}^{f_{1} x} & P_{k-1}^{f_{1}}
\end{array}\right]\left[\begin{array}{c}
A_{k-1}^{T} \\
F_{1, k-1}^{x T}
\end{array}\right]+Q_{k-1} .
\end{aligned}
$$

Here $P_{k-1}^{x f_{1}}$ and $P_{k-1}^{f_{1}}$ will be defined later.

Note that problem (19) can be written more compactly in the form (1) and (2) with the identifications

$$
\begin{aligned}
x & \longleftarrow \operatorname{col}\left(x_{k}-\hat{x}_{k / k-1}, f_{1, k / k}, f_{2, k-1 / k}\right), \\
b & \longleftarrow\left[\begin{array}{c}
y_{k}-C_{k} \widehat{x}_{k / k-1} \\
0
\end{array}\right], \\
A & \longleftarrow\left[\begin{array}{ccc}
C_{k} & F_{1, k}^{y} & 0 \\
I & 0 & -F_{2, k-1}^{x}
\end{array}\right], \\
\delta A & \longleftarrow\left[\begin{array}{ccc}
\Delta C_{k} & 0 & 0 \\
0 & 0 & 0
\end{array}\right], \\
\delta b & \longleftarrow\left[\begin{array}{c}
-\Delta C_{k} \widehat{x}_{k / k-1} \\
0
\end{array}\right], \\
W & \\
& \longleftarrow \operatorname{diag}\left(R_{k}^{-1}, \bar{P}_{k}^{-1}\right),
\end{aligned}
$$

$$
\begin{aligned}
& H \longleftarrow\left[\begin{array}{cc}
H_{2, k} & 0 \\
0 & 0
\end{array}\right], \\
& N_{a} \longleftarrow\left[\begin{array}{ccc}
E_{k} & 0 & 0 \\
0 & 0 & 0
\end{array}\right], \\
& N_{b} \longleftarrow\left[\begin{array}{c}
-E_{k} \widehat{x}_{k / k-1} \\
0
\end{array}\right], \\
& \Delta \longleftarrow F_{k} .
\end{aligned}
$$

From (1)-(3) the solution to (19) is given by

$$
\begin{aligned}
& {\left[\begin{array}{c}
\widehat{x}_{k}-\widehat{x}_{k / k-1} \\
\hat{f}_{1, k / k} \\
\hat{f}_{2, k-1 / k}
\end{array}\right]} \\
& =\left[\begin{array}{ccc}
\breve{P}_{k}^{-1} & C_{k}^{T} \bar{R}_{k}^{-1} F_{1, k}^{y} & -\bar{P}_{k}^{-1} F_{2, k-1}^{x} \\
F_{1, k}^{y T} \bar{R}_{k}^{-1} C_{k} & \breve{D}_{1, k}^{-1} & 0 \\
-F_{2, k-1}^{x T} \bar{P}_{k}^{-1} & 0 & \breve{D}_{2, k-1}^{-1}
\end{array}\right]^{-1} \\
& \cdot\left[\begin{array}{c}
C_{k}^{T} \bar{R}_{k}^{-1}\left(y_{k}-C_{k} \widehat{x}_{k / k-1}\right)-\hat{\lambda}_{k} E_{k}^{T} E_{k} \widehat{x}_{k / k-1} \\
F_{1, k}^{y T} \bar{R}_{k}^{-1}\left(y_{k}-C_{k} \widehat{x}_{k / k-1}\right)
\end{array}\right],
\end{aligned}
$$

where

$$
\begin{aligned}
\breve{P}_{k}^{-1} & =\bar{P}_{k}^{-1}+C_{k}^{T} \bar{R}_{k}^{-1} C_{k}, \\
\breve{D}_{1, k}^{-1} & =F_{1, k}^{y T} \bar{R}_{k}^{-1} F_{1, k}^{y},
\end{aligned}
$$




$$
\begin{aligned}
\breve{D}_{2, k-1}^{-1} & =F_{2, k-1}^{x T} \bar{P}_{k}^{-1} F_{2, k-1}^{x}, \\
\bar{R}_{k}^{-1} & =\left(R_{k}-\hat{\lambda}_{k}^{-1} H_{2, k} H_{2, k}^{T}\right)^{-1} .
\end{aligned}
$$

Moreover, $\hat{\lambda}_{k}$ is the minimizing argument in the interval $\hat{\lambda}_{k}>\left\|H_{2, k}^{T} R_{k}^{-1} H_{2, k}\right\|=\lambda_{l, k}$ of the corresponding scalar-valued function $G(\lambda)$ in (6) constructed with identification (21).

Using [28, Prop. 2.8.7], we find that the inverse in (22) can be written as

$$
\begin{aligned}
& {\left[\begin{array}{ccc}
\breve{P}_{k}^{-1} & C_{k}^{T} \bar{R}_{k}^{-1} F_{1, k}^{y} & -\bar{P}_{k}^{-1} F_{2, k-1}^{x} \\
F_{1, k}^{y T} \bar{R}_{k}^{-1} C_{k} & \breve{D}_{1, k}^{-1} & 0 \\
-F_{2, k-1}^{x T} \bar{P}_{k}^{-1} & 0 & \breve{D}_{2, k-1}^{-1}
\end{array}\right]} \\
& =\left[\begin{array}{ccc}
P_{k} & P_{k}^{x f_{1}} & P_{k}^{x f_{2}} \\
P_{k}^{f_{1} x} & P_{k}^{f_{1}} & P_{k}^{f_{1} f_{2}} \\
P_{k}^{f_{2} x} & P_{k}^{f_{2} f_{1}} & P_{k-1}^{f_{2}}
\end{array}\right], \\
& P_{k}^{x f_{1}}=\left(P_{k}^{f_{1} x}\right)^{T}=-P_{k} C_{k}^{T} \bar{R}_{k}^{-1} F_{1, k}^{y} \breve{D}_{1, k}^{-1}, \\
& P_{k}^{x f_{2}}=\left(P_{k}^{f_{2} x}\right)^{T}=P_{k} \bar{P}_{k}^{-1} F_{2, k-1}^{x} \breve{D}_{2, k-1}^{-1}, \\
& P_{k}^{f_{1} f_{2}}=\left(P_{k}^{f_{2} f_{1}}\right)^{T} \\
& =-\breve{D}_{1, k} F_{1, k}^{y T} \bar{R}_{k}^{-1} C_{k} P_{k} \bar{P}_{k}^{-1} F_{2, k-1}^{x} \breve{D}_{2, k-1}^{-1} .
\end{aligned}
$$

Note that $P_{k}, P_{k}^{f_{1}}$, and $P_{k-1}^{f_{2}}$ can be identified as the covariance matrices of $\hat{x}_{k / k}, \widehat{f}_{1, k}$, and $\hat{f}_{2, k-1}$; that is,

$$
\begin{aligned}
& P_{k}=E\left[\left(x_{k}-\hat{x}_{k / k}\right)\left(x_{k}-\hat{x}_{k / k}\right)^{T}\right], \\
& P_{k}^{f_{1}}=E\left[\left(f_{1, k / k}-\hat{f}_{1, k / k}\right)\left(f_{1, k / k}-\hat{f}_{1, k / k}\right)^{T}\right], \\
& P_{k-1}^{f_{2}} \\
& \quad=E\left[\left(f_{2, k-1 / k}-\hat{f}_{2, k-1 / k}\right)\left(f_{2, k-1 / k}-\widehat{f}_{2, k-1 / k}\right)^{T}\right],
\end{aligned}
$$

where the inverse of $P_{k}, P_{k}^{f_{1}}$, and $P_{k-1}^{f_{2}}$ are given by

$$
\begin{gathered}
P_{k}^{-1}=\breve{P}_{k, 1}^{-1}-C_{k}^{T} \bar{R}_{k}^{-1} F_{1, k}^{y} \breve{D}_{1, k} F_{1, k}^{y T} \bar{R}_{k}^{-1} C_{k}, \\
\left(P_{k}^{f_{1}}\right)^{-1}=\breve{D}_{1, k}^{-1}-F_{1, k}^{y T} \bar{R}_{k}^{-1} C_{k} \breve{P}_{k, 1} C_{k}^{T} \bar{R}_{k}^{-1} F_{1, k}^{y},
\end{gathered}
$$

$$
\begin{aligned}
\left(P_{k-1}^{f_{2}}\right)^{-1} & =\breve{D}_{2, k-1}^{-1}-F_{2, k-1}^{x T} \bar{P}_{k}^{-1} \widehat{P}_{k, 1} \bar{P}_{k}^{-1} F_{2, k-1}^{x}, \\
\breve{P}_{k, 1}^{-1} & =\breve{P}_{k}^{-1}-\bar{P}_{k}^{-1} F_{2, k-1}^{x} \breve{D}_{2, k-1} F_{2, k-1}^{x T} \bar{P}_{k}^{-1} \\
\widehat{P}_{k, 1}^{-1} & =\breve{P}_{k}^{-1}-C_{k}^{T} \bar{R}_{k}^{-1} F_{1, k}^{y} \breve{D}_{1, k} F_{1, k}^{y T} \bar{R}_{k}^{-1} C_{k} .
\end{aligned}
$$

Finally substituting (24) in (22) yields

$$
\begin{aligned}
\hat{x}_{k / k}= & \left(I-\widehat{\lambda}_{k} P_{k} E_{k}^{T} E_{k}\right) \hat{x}_{k / k-1} \\
& +P_{k} C_{k}^{T} \bar{R}_{k}^{-1}\left(y_{k}-C_{k} \widehat{x}_{k / k-1}-F_{1, k}^{y} \widehat{f}_{1, k / k}\right), \\
\widehat{f}_{1, k / k}= & M_{k}^{f_{1}}\left(y_{k}-\bar{C}_{k} \widehat{x}_{k / k-1}\right), \\
\widehat{f}_{2, k-1 / k}= & M_{k}^{f_{2}}\left(y_{k}-\bar{C}_{k} \widehat{x}_{k / k-1}\right),
\end{aligned}
$$

where

$$
\begin{aligned}
M_{k}^{f_{1}} & =\left(F_{1, k}^{y T} \widetilde{R}_{1, k}^{-1} F_{1, k}^{y}\right)^{-1} F_{1, k}^{y T} \widetilde{R}_{1, k}^{-1}, \\
M_{k}^{f_{2}} & =\left(F_{2, k-1}^{x T} \widetilde{R}_{2, k}^{-1} F_{2, k-1}^{x}\right)^{-1} F_{2, k-1}^{x T} \widetilde{R}_{2, k}^{-1}, \\
\bar{C}_{k} & =C_{k}-\widehat{\lambda}_{k} C_{k} \bar{P}_{k} E_{k}^{T} E_{k}, \\
\widetilde{R}_{1, k} & =C_{k} \widetilde{P}_{k} C_{k}^{T}+\bar{R}_{k}, \\
\widetilde{R}_{2, k}^{-1} & =\bar{P}_{k}^{-1}-\bar{P}_{k}^{-1} \widehat{P}_{k, 1} \bar{P}_{k}^{-1}, \\
\widetilde{P}_{k}^{-1} & =\bar{P}_{k}^{-1}-\bar{P}_{k}^{-1} F_{2, k-1}^{x} \breve{D}_{2, k-1} F_{2, k-1}^{x T} \bar{P}_{k}^{-1} .
\end{aligned}
$$

Furthermore, we find that $P_{k}^{f_{1}}$ and $P_{k-1}^{f_{2}}$ are given, respectively, by

$$
\begin{aligned}
P_{k}^{f_{1}} & =\left(F_{1, k}^{y T} \widetilde{R}_{k}^{-1} F_{1, k}^{y}\right)^{-1}, \\
P_{k-1}^{f_{2}} & =\left(F_{2, k-1}^{x T} \widetilde{R}_{2, k}^{-1} F_{2, k-1}^{x}\right)^{-1} .
\end{aligned}
$$

Finally, it follows from (17) and (30) that

$$
\begin{aligned}
\hat{x}_{k+1 / k}= & A_{p, k} \widehat{x}_{k / k-1}+F_{1, k}^{x} \widehat{f}_{1, k / k}+B_{k} u_{k} \\
& +K_{p, k}\left(y_{k}-C_{k} \widehat{x}_{k / k-1}-F_{1, k}^{y} \widehat{f}_{1, k / k}\right),
\end{aligned}
$$

where $A_{p, k}$ and $K_{p, k}$ are defined in terms of the parameter $P_{k}$ as

$$
\begin{aligned}
& A_{p, k}=A_{k}\left(I-\hat{\lambda}_{k} P_{k} E_{k}^{T} E_{k}\right), \\
& K_{p, k}=A_{k} P_{k} C_{k}^{T} \bar{R}_{k}^{-1} .
\end{aligned}
$$


Note that these expressions for $A_{p, k}$ and $K_{p, k}$ are defined linearly in terms of $P_{k}$ and have been determined by assuming uncertainties in $C_{k}$ alone. We will incorporate the uncertainties in $A_{k}$ in the next section.

4.2. Bounded Uncertainties in $A_{k}$ and $C_{k}$. We now incorporate uncertainties into $A_{k}$. That is, we consider norm bounded uncertainties in $A_{k}$ and $C_{k}$ as in (13) and (15). We will move to select the parameter $P_{k}$ by assuming uncertainties in $A_{k}$ alone and one that meets robustness criterion (19) when there are uncertainties in $C_{k}$.

Denoting $\tilde{x}_{k}=x_{k}-\widehat{x}_{k / k-1}$, we define the extended weight vector $\zeta_{k}=\left[\begin{array}{c}\tilde{x}_{k} \\ \hat{x}_{k / k-1}\end{array}\right]$. Then in the absence of uncertainties in $C_{k}$, we find that $\zeta_{k}$ satisfies

$$
\zeta_{k+1}=\left(\bar{A}_{k, c}+\bar{H}_{k, c} F_{k} \bar{E}_{k, c}\right) \zeta_{k}+\bar{F}_{k, c} \bar{f}_{k, c}+\bar{G}_{k, c} \bar{w}_{k}
$$

where

$$
\begin{aligned}
\bar{A}_{k, c} & =\left(\begin{array}{cc}
A_{k}-K_{p, k} C_{k} & A_{k}-A_{p, k} \\
K_{p, k} C_{k} & A_{p, k}
\end{array}\right), \\
\bar{F}_{k, c} & =\left[\begin{array}{ccc}
F_{k}^{x} & -K_{p, k} F_{1, k}^{y} & 0 \\
0 & K_{p, k} F_{1, k}^{y} & F_{1, k}^{x}
\end{array}\right], \\
\bar{G}_{k, c} & =\left[\begin{array}{ccc}
I & -K_{p, k} & F_{2, k}^{x} \\
0 & K_{p, k} & 0
\end{array}\right], \\
\bar{H}_{k, c} & =\left(\begin{array}{cc}
H_{1, k} & 0 \\
0 & 0
\end{array}\right), \\
\bar{E}_{k, c} & =\left(\begin{array}{cc}
E_{k} & E_{k} \\
0 & 0
\end{array}\right), \\
\bar{f}_{k, c} & =\left(\begin{array}{c}
\tilde{f}_{k} \\
\tilde{f}_{1, k} \\
\hat{f}_{1, k}
\end{array}\right), \\
\bar{w}_{k} & =\left(\begin{array}{c}
w_{k} \\
v_{k} \\
\hat{f}_{2, k}
\end{array}\right) .
\end{aligned}
$$

The covariance matrix of $\zeta_{k}$ satisfies

$$
\begin{aligned}
\Sigma_{k+1} & \\
= & E\left\{\left(\bar{A}_{k, c}+\bar{H}_{k, c} F_{k} \bar{E}_{k, c}\right) \Sigma_{k}\left(\bar{A}_{k, c}+\bar{H}_{k, c} F_{k} \bar{E}_{k, c}\right)^{T}\right\} \\
& +E\left\{\left(\bar{A}_{k, c}+\bar{H}_{k, c} F_{k} \bar{E}_{k, c}\right) \Gamma_{k} \bar{F}_{k, c}^{T}\right\} \\
& +E\left\{\bar{F}_{k, c} \Gamma_{k}^{T}\left(\bar{A}_{k}+\bar{H}_{k} F_{k} \bar{E}_{k, c}\right)^{T}\right\}+\bar{F}_{k, c} \gamma_{k} \bar{F}_{k, c}^{T} \\
& +\bar{G}_{k, c} S_{k} \bar{G}_{k, c}^{T}
\end{aligned}
$$

where

$$
\begin{aligned}
S_{k} & =\left(\begin{array}{ccc}
Q_{k} & 0 & 0 \\
0 & R_{k} & 0 \\
0 & 0 & P_{k}^{f_{2}}
\end{array}\right), \\
\Gamma_{k} & =\left(\begin{array}{ccc}
P_{k}^{x f} & P_{k}^{x f_{1}} & 0 \\
0 & 0 & 0 \\
0 & 0 & 0
\end{array}\right), \\
\Upsilon_{k} & =\left(\begin{array}{ccc}
P_{k}^{f} & P_{k}^{f f_{1}} & 0 \\
P_{k}^{f_{1} f} & P_{k}^{f_{1}} & 0 \\
0 & 0 & P_{k}^{f_{1}}
\end{array}\right),
\end{aligned}
$$

where

$$
\begin{aligned}
& P_{k}^{f}=\left[\begin{array}{ll}
V_{1, k} & V_{2, k}
\end{array}\right]\left[\begin{array}{cc}
P_{k}^{f_{1}} & P_{k}^{f_{1} f_{2}} \\
P_{k}^{f_{2} f_{1}} & P_{k}^{f_{2}}
\end{array}\right]\left[\begin{array}{c}
V_{1, k}^{T} \\
V_{2, k}^{T}
\end{array}\right], \\
& P_{k}^{x f}=V_{1, k} P_{k}^{x f_{1}}+V_{2, k} P_{k}^{x f_{2}}, \\
& P_{k}^{f f_{1}}=\left(P_{k}^{f_{1} f}\right)^{T}=V_{1} P_{k}^{f_{1}}+V_{2} \breve{F}_{2, k}^{-1} P_{k}^{f_{2} f_{1}} .
\end{aligned}
$$

Now observe that the expressions for $\left\{A_{p, k}, K_{p, k}\right\}$ are parameterized linearly in terms of the parameter $P_{k}$. We shall choose $P_{k}$ by minimizing an upper bound of $\Sigma_{k+1}$ in the absence of uncertainties in $C_{k}$.

Let $\alpha_{k}$ be a scalar such that $\alpha_{k} I-\bar{E}_{c, k} \Sigma_{k} \bar{E}_{c, k}^{T}>0$ and let $\beta_{k}$ be a scalar such that $\beta_{k} I-\bar{E}_{c, k} \Upsilon_{k} \bar{E}_{c, k}^{T}>0$. Bearing in mind (45), we can see that $\Sigma_{k+1}$ is bounded by

$$
\begin{aligned}
\Sigma_{k+1} \geq & \bar{A}_{k, c} \Sigma_{k} \bar{A}_{k, c}^{T}+\bar{A}_{k, c} \Gamma_{k} \bar{F}_{k, c}^{T}+\bar{F}_{k, c} \Gamma_{k}^{T} \bar{A}_{k, c}^{T} \\
& +\bar{F}_{k, c} \Upsilon_{k} \bar{F}_{k, c}^{T}+\bar{G}_{k, c} S_{k} \bar{G}_{k, c}^{T} \\
& +\left(\alpha_{k}+\beta_{k}\right) \bar{H}_{k, c} \bar{H}_{k, c}^{T} .
\end{aligned}
$$

We choose $P_{k}>0$, by solving

$$
\min _{P_{k}>0} \operatorname{tr}\left(\Sigma_{k+1}\right),
$$

subject to $\Sigma_{k+1}$

$$
\begin{aligned}
\geq & \bar{A}_{k, c} \Sigma_{k} \bar{A}_{k, c}^{T}+\bar{A}_{k, c} \Gamma_{k} \bar{F}_{k, c}^{T}+\bar{F}_{k, c} \Gamma_{k}^{T} \bar{A}_{k, c}^{T} \\
& +\bar{F}_{k, c} \Upsilon_{k} \bar{F}_{k, c}^{T}+\bar{G}_{k, c} S_{k} \bar{G}_{k, c}^{T} \\
& +\left(\alpha_{k}+\beta_{k}\right) \bar{H}_{k, c} \bar{H}_{k, c}^{T} .
\end{aligned}
$$


Or equivalently

$$
\left[\begin{array}{cccc}
-\Sigma_{k+1}+\bar{A}_{k, c} \Gamma_{k} \bar{F}_{k, c}^{T}+\bar{F}_{k, c} \Gamma_{k}^{T} \bar{A}_{k, c}^{T}+\left(\alpha_{k}+\beta_{k}\right) \bar{H}_{k, c} \bar{H}_{k, c}^{T} & \bar{A}_{k, c} \Sigma_{k} & \bar{G}_{k, c} S_{k}^{1 / 2} & \bar{F}_{k, c} \Upsilon_{k} \\
\Sigma_{k} \bar{A}_{k, c}^{T} & -\Sigma_{k} & 0 & 0 \\
S_{k} \bar{G}_{k, c}^{T / 2} & 0 & -I & 0 \\
\Upsilon_{k} \bar{F}_{k, c}^{T} & 0 & 0 & -\Upsilon_{k}
\end{array}\right] \leq 0 .
$$

Since inequality (51) is affine in $A_{k}, P_{k}$ thus found will ensure minimum error covariance $\Sigma_{k}$ over all possible $A_{k}$ in the bounded domain. Therefore the desired robust proportional filter is given by (40)-(41) and (31)-(38). So initializing $\Sigma_{0}=$ $\operatorname{diag}\left\{\varepsilon I, P_{0}\right\}$ for $P_{0}>0$ and a scalar $\varepsilon>0$, the resulting filter is listed in the following section.

4.3. Summary of the RPF. In this section we summarize the filter equations, we assume that $\hat{x}_{0}$ is the estimate of the initial state a zero-mean and has known variance $P_{0}$.

Initial Condition. $\widehat{x}_{0}=0, \Sigma_{0}=\operatorname{diag}\left\{\varepsilon I, P_{0}\right\}$, where $P_{0}>0$ and $\varepsilon>0$.

Step 1. If $H_{2, k}=0$, then set $\widehat{\lambda}_{k}=0$. Otherwise, construct $G(\lambda)$ of (6) with identification (21) and determine $\widehat{\lambda}_{k}$ by minimizing $G(\lambda)$ over the interval

$$
\hat{\lambda}_{k}>\lambda_{l, k}=\left\|H_{2, k}^{T} R_{k}^{-1} H_{2, k}\right\| .
$$

Step 2. Using $\Sigma_{k}$, compute $\left\{P_{k}, \Sigma_{k+1}\right\}$ by solving (49) subject to inequality (51), where $\left\{\bar{A}_{k, c}, \bar{F}_{k, c}, \bar{G}_{k, c}, \bar{H}_{k, c}\right\}$ and $\left\{S_{k}, \Gamma_{k}\right.$, $\left.\Upsilon_{k}\right\}$ are given, respectively, by (43) and (46).

Step 3. Robust simultaneous fault and state estimation are as follows:

$$
\begin{aligned}
\widehat{f}_{1, k / k} & =M_{k}^{f_{1}}\left(y_{k}-\bar{C}_{k} \widehat{x}_{k / k-1}\right), \\
\widehat{f}_{2, k-1 / k} & =M_{k}^{f_{2}}\left(y_{k}-\bar{C}_{k} \widehat{x}_{k / k-1}\right), \\
\widehat{f}_{2, k} & =\breve{F}_{2, k}^{x} \hat{\bar{f}}_{2, k}, \\
\widehat{f}_{k} & =V_{1, k} \widehat{f}_{1, k}+V_{2, k} \hat{\bar{f}}_{2, k},
\end{aligned}
$$

where $M_{k}^{f_{1}}, M_{k}^{f_{2}}$, and $\bar{C}_{k}$ are given by (33)-(37).

$$
\begin{aligned}
& \text { Update } \widehat{x}_{k / k-1} \text { to } \widehat{x}_{k+1 / k} \text { as } \\
& \begin{aligned}
\hat{x}_{k+1 / k}= & A_{p, k} \widehat{x}_{k / k-1}+F_{1, k}^{x} \widehat{f}_{1, k / k}+B_{k} u_{k} \\
& +K_{p, k}\left(y_{k}-C_{k} \widehat{x}_{k / k-1}-F_{k, 1}^{y} \widehat{f}_{1, k / k}\right),
\end{aligned}
\end{aligned}
$$

where $A_{p, k}$ and $K_{p, k}$ are given by (41).
Remark 1. Note that the robust filter (RPF) developed in the previous section gives a better estimation of the state and the fault; however, when the unknown fault is timevarying, the performances of the robust filter can deteriorate. So we will extend the RPF to further propose a new robust proportional integral filter (RPIF) structure, in which the integral action is believed to improve robust estimation of the unknown time-varying faults and to improve robustness against uncertainties.

\section{Robust Proportional Integral Filter Design}

In this section, we propose to design a new robust proportional integral filter (RPIF) for stochastic linear uncertain system (13) and (15) to improve the estimation of unknown time-varying faults and to improve robustness against uncertainties such as disturbances and modeling errors.

The proposed filter has the following structure:

$$
\begin{aligned}
\widehat{x}_{k+1 / k}= & A_{p, k} \widehat{x}_{k / k-1}+F_{1, k}^{x} \hat{f}_{1, k / k}+B_{k} u_{k} \\
& +K_{p, k}\left(y_{k}-C_{k} \widehat{x}_{k / k-1}-F_{1, k}^{y} \widehat{f}_{1, k / k}\right) \\
& +K_{z, k} z_{k}, \\
z_{k+1}= & \alpha_{0} z_{k}+H_{k}\left(y_{k}-C_{k} \widehat{x}_{k / k-1}-F_{1, k}^{y} \widehat{f}_{1, k / k}\right), \\
\widehat{f}_{1, k / k}= & M_{k}^{f_{1}}\left(y_{k}-\bar{C}_{k} \widehat{x}_{k / k-1}\right), \\
\widehat{f}_{2, k-1 / k}= & M_{k}^{f_{2}}\left(y_{k}-\bar{C}_{k} \widehat{x}_{k / k-1}\right), \\
\widehat{f}_{k / k}= & V_{1, k} \hat{f}_{1, k / k}+V_{2, k} \hat{\bar{f}}_{2, k / k}, \\
\widehat{f}_{2, k}= & \breve{F}_{2, k}^{x} \hat{\bar{f}}_{2, k},
\end{aligned}
$$

where the matrices $K_{p, k}$ and $K_{z, k}$ represent a proportional gain and an integral gain, respectively. The variable $z_{k}$ is 
related to the weighted integral of the output estimation error. The constant value $\alpha_{0}$ stands for a fading effect coefficient that regulates the transient response. The matrix $H_{k}$ is an integral effect coefficient. The two design parameters are assumed to be preselected by designers. Theses expressions for $A_{p, k}, K_{p, k}, K_{z, k}, M_{k}^{f_{1}}$, and $M_{k}^{f_{2}}$ have been determined by assuming uncertainties in $C_{k}$ alone. We now move on to select the parameter $P_{k}$ by assuming uncertainties in $A_{k}$ alone. By doing so, we will arrive at a filter that minimizes a bound on the state error covariance matrix when there are uncertainties in $A_{k}$ alone.

Proceeding in the same manner as in the previous section, we know that the expression for $\left\{A_{p, k}, K_{p, k}, M_{k}^{f_{1}}, M_{k}^{f_{2}}\right\}$ can be parameterized linearly in terms of the parameter $P_{k}$.

Defining the extended weight vector $\eta_{k}=\left(\begin{array}{l}\tilde{x}_{k} \\ z_{k}\end{array}\right)$. Ignoring the uncertainties in $C_{k}$, we find that $\eta_{k}$ satisfies

$$
\begin{aligned}
\eta_{k+1}= & \left(\bar{A}_{k}+\bar{H}_{k} F_{k} \bar{E}_{1, k}\right) \eta_{k}+\left(\bar{F}_{k}+\bar{H}_{k} F_{k} \bar{E}_{2, k}\right) \bar{f}_{k} \\
& +\bar{G}_{k} \bar{w}_{k},
\end{aligned}
$$

where

$$
\begin{aligned}
\bar{A}_{k} & =\left(\begin{array}{ccc}
A_{k}-K_{p, k} C_{k} & -K_{z, k} \\
H_{k} C_{k} & \alpha I
\end{array}\right), \\
\bar{F}_{k} & =\left[\begin{array}{ccc}
A_{k}-A_{p, k} & F_{k}^{x} & -K_{p, k} F_{1, k}^{y} \\
0 & 0 & H_{k} F_{1, k}^{y}
\end{array}\right], \\
\bar{G}_{k} & =\left[\begin{array}{ccc}
I & -K_{p, k} & F_{2, k}^{x} \\
0 & H_{k} & 0
\end{array}\right], \\
\bar{H}_{k} & =\left(\begin{array}{cc}
H_{1, k} & 0 \\
0 & 0
\end{array}\right), \\
\bar{E}_{1, k} & =\left(\begin{array}{cc}
E_{k} & 0 \\
0 & 0
\end{array}\right), \\
\bar{E}_{2, k} & =\left[\begin{array}{cc}
E_{k} & 0 \\
0 & 0 \\
\hat{x}_{k / k-1}
\end{array}\right], \\
\bar{f}_{k} & =\left(\begin{array}{c}
\tilde{f}_{k} \\
\tilde{f}_{k, 1}
\end{array}\right), \\
\bar{w}_{k} & =\left(\begin{array}{c}
w_{k} \\
v_{k} \\
\hat{f}_{2, k}
\end{array}\right) .
\end{aligned}
$$

Suppose that $\bar{\Sigma}_{k+1}$ satisfies

$$
\begin{aligned}
\bar{\Sigma}_{k+1}= & E\left\{\left(\bar{A}_{k}+\bar{H}_{k} F_{k} \bar{E}_{1, k}\right) \bar{\Sigma}_{k}\left(\bar{A}_{k}+\bar{H}_{k} F_{k} \bar{E}_{1, k}\right)^{T}\right\} \\
& +E\left\{\left(\bar{A}_{k}+\bar{H}_{k} F_{k} \bar{E}_{1, k}\right) \bar{\Gamma}_{k}\left(\bar{F}_{k}+\bar{H}_{k} F_{k} \bar{E}_{2, k}\right)^{T}\right\}
\end{aligned}
$$

$$
\begin{aligned}
& +E\left\{\left(\bar{F}_{k}+\bar{H}_{k} F_{k} \bar{E}_{2, k}\right) \bar{\Gamma}_{k}^{T}\left(\bar{A}_{k}+\bar{H}_{k} F_{k} \bar{E}_{1, k}\right)^{T}\right\} \\
& +E\left\{\left(\bar{F}_{k}+\bar{H}_{k} F_{k} \bar{E}_{2, k}\right) \bar{\Upsilon}_{k}\left(\bar{F}_{k}+\bar{H}_{k} F_{k} \bar{E}_{2, k}\right)^{T}\right\} \\
& +\bar{G}_{k} S_{k} \bar{G}_{k}^{T},
\end{aligned}
$$

where

$$
\begin{aligned}
S_{k} & =\left(\begin{array}{ccc}
Q_{k} & 0 & 0 \\
0 & R_{k} & 0 \\
0 & 0 & P_{k}^{f_{2}}
\end{array}\right), \\
\bar{\Gamma}_{k} & =\left(\begin{array}{ccc}
0 & P_{k}^{x f} & P_{k}^{x f_{1}} \\
0 & P_{k}^{z f} & P_{k}^{z f_{1}} \\
0 & 0 & 0
\end{array}\right),
\end{aligned}
$$

$$
\begin{aligned}
& \bar{\Upsilon}_{k}=\left(\begin{array}{ccc}
P_{k}^{x}-P_{k} & 0 & 0 \\
0 & P_{k}^{f} & P_{k}^{f f_{1}} \\
0 & P_{k}^{f_{1} f} & P_{k}^{f_{1}}
\end{array}\right), \\
& P_{k}^{z f}=H_{k}\left(C_{k} P_{k}^{x f}+F_{1, k}^{y} P_{k}^{f_{1}} V_{1}^{T}+F_{1, k}^{y} P_{k}^{f_{1} f_{2}} \breve{F}_{2, k}^{-T} V_{2}^{T}\right), \\
& P_{k}^{z f_{1}}=H_{k}\left(C_{k} P_{k}^{x f_{1}}+F_{1, k}^{y} P_{k}^{f_{1}}\right) .
\end{aligned}
$$

Let $\bar{\alpha}_{k}$ be a scalar such that $\bar{\alpha}_{k} I-\bar{E}_{1, k} \bar{\Sigma}_{k} \bar{E}_{1, k}^{T}>0$ and let $\bar{\beta}_{k}$ be a scalar such that $\bar{\beta}_{k} I-\bar{E}_{2, k} \bar{\Upsilon}_{k} \bar{E}_{2, k}^{T}>0$. Bearing in mind (59), we can see that $\bar{\Sigma}_{k+1}$ is bounded by

$$
\begin{gathered}
\bar{\Sigma}_{k+1} \geq \bar{A}_{k} \bar{\Sigma}_{k} \bar{A}_{k}^{T}+\bar{A}_{k} \bar{\Gamma}_{k} \bar{F}_{k}^{T}+\bar{F}_{k} \bar{\Gamma}_{k}^{T} \bar{A}_{k}^{T}+\bar{F}_{k} \bar{\Upsilon}_{k} \bar{F}_{k}^{T} \\
+\bar{G}_{k} S_{k} \bar{G}_{k}^{T}+\left(\bar{\alpha}_{k}+\bar{\beta}_{k}\right) \bar{H}_{k} \bar{H}_{k}^{T} .
\end{gathered}
$$

We choose $P_{k}>0$, by solving

$$
\min _{P_{k}>0} \operatorname{tr}\left(\bar{\Sigma}_{k+1}\right),
$$

subject to $\bar{\Sigma}_{k+1}$

$$
\begin{aligned}
\geq & \bar{A}_{k} \bar{\Sigma}_{k} \bar{A}_{k}^{T}+\bar{A}_{k} \bar{\Gamma}_{k} \bar{F}_{k}^{T}+\bar{F}_{k} \bar{\Gamma}_{k}^{T} \bar{A}_{k}^{T} \\
& +\bar{F}_{k} \bar{\Upsilon}_{k} \bar{F}_{k}^{T}+\bar{G}_{k} S_{k} \bar{G}_{k}^{T} \\
& +\left(\bar{\alpha}_{k}+\bar{\beta}_{k}\right) \bar{H}_{k} \bar{H}_{k}^{T} .
\end{aligned}
$$


Or equivalently

$$
\left[\begin{array}{cccc}
-\bar{\Sigma}_{k+1}+\bar{A}_{k} \bar{\Gamma}_{k} \bar{F}_{k}^{T}+\bar{F}_{k} \bar{\Gamma}_{k}^{T} \bar{A}_{k}^{T}+\left(\bar{\alpha}_{k}+\bar{\beta}_{k}\right) \bar{H}_{k} \bar{H}_{k}^{T} & \bar{A}_{k} \bar{\Sigma}_{k} & \bar{G}_{k} S_{k}^{1 / 2} & \bar{F}_{k} \bar{\Upsilon} \\
\bar{\Sigma}_{k} \bar{A}_{k}^{T} & -\bar{\Sigma}_{k} & 0 & 0 \\
S_{k} \bar{G}_{k}^{T / 2} & 0 & -I & 0 \\
\bar{\Upsilon}_{k} \bar{F}_{k}^{T} & 0 & 0 & -\bar{\Upsilon}_{k}
\end{array}\right] \leq 0 .
$$

Therefore the desired robust proportional integral filter (RPIF) is given by (55) where $P_{k}$ is the positive-definite solution of (66) subject to (67) with the initial condition $\bar{\Sigma}_{0}=$ $\operatorname{diag}\left\{P_{0}, \varepsilon I\right\}$ for $P_{0}>0$. Note that there always exists a solution to (66)-(67). The resulting filter is listed in following section.

Summary of the RPIF. In this section we summarize the filter equations; we assume that $\widehat{x}_{0}$ is the estimate of the initial state a zero-mean and has known variance $P_{0}$.

The initialization step of the filter is then given as follows.

Initial Condition. $\widehat{x}_{0}=0, \bar{\Sigma}_{0}=\operatorname{diag}\left\{P_{0}, \varepsilon I\right\}$, where $P_{0}>$ 0 and $\varepsilon>0$.

Step 1. If $H_{2, k}=0$, then set $\widehat{\lambda}_{k}=0$. Otherwise, construct $G(\lambda)$ of (6) with the identification (21) and determine $\hat{\lambda}_{k}$ by minimizing $G(\lambda)$ over the interval

$$
\hat{\lambda}_{k}>\lambda_{l, k}=\left\|H_{2, k}^{T} R_{k}^{-1} H_{2, k}\right\| .
$$

Step 2. Using $\bar{\Sigma}_{k}$, compute $\left\{P_{k}, K_{z, k}, \bar{\Sigma}_{k+1}\right\}$ by solving (66) subject to inequality (67), where $\left\{\bar{A}_{k}, \bar{F}_{k}, \bar{G}_{k}, \bar{H}_{k}\right\}$ and $\left\{S_{k}, \bar{\Gamma}_{k}, \bar{\Upsilon}_{k}\right\}$ are given by (57) and (60)-(62).

Step 3. Robust simultaneous fault and state estimation are as follows:

$$
\begin{aligned}
\widehat{f}_{1, k / k} & =M_{k}^{f_{1}}\left(y_{k}-\bar{C}_{k} \widehat{x}_{k / k-1}\right), \\
\widehat{f}_{2, k-1 / k} & =M_{k}^{f_{2}}\left(y_{k}-\bar{C}_{k} \widehat{x}_{k / k-1}\right), \\
\widehat{f}_{2, k} & =\breve{F}_{2, k}^{x} \hat{\bar{f}}_{2, k}, \\
\widehat{f}_{k} & =V_{1, k} \widehat{f}_{1, k}+V_{2, k} \hat{\bar{f}}_{2, k},
\end{aligned}
$$

where $M_{k}^{f_{1}}, M_{k}^{f_{2}}$, and $\bar{C}_{k}$ are given by (33)-(35).

Update $\hat{x}_{k / k-1}$ to $\hat{x}_{k+1 / k}$ as

$$
\begin{aligned}
\widehat{x}_{k+1 / k}= & A_{p, k} \widehat{x}_{k / k-1}+F_{1, k}^{x} \widehat{f}_{1, k / k}+B_{k} u_{k} \\
& +K_{p, k}\left(y_{k}-C_{k} \widehat{x}_{k / k-1}-F_{k, 1}^{y} \widehat{f}_{1, k / k}\right) \\
& +K_{z, k} z_{z},
\end{aligned}
$$

$$
z_{k+1}=\alpha_{0} z_{k}+H_{k}\left(y_{k}-C_{k} \widehat{x}_{k / k-1}-F_{k, 1}^{y} \widehat{f}_{1, k / k}\right),
$$

where $A_{p, k}$ and $K_{p, k}$ are given by (41).

\section{Illustrative Example}

Robust estimation of simultaneous actuator and sensor faults is as follows.

In this section, we propose the use of the resulting filters RPF and RPIF to solve the robust estimation of simultaneous actuator and sensor faults problem.

We consider the same numerical example used in (Chen and Patton [5, 6]). The linearized model of a simplified longitudinal flight control system is as follows:

$$
\begin{aligned}
x_{k+1} & =\left(A_{k}+\Delta A_{k}\right) x_{k}+B_{k} u_{k}+F_{k}^{a} f_{k}^{a}+w_{k}, \\
y_{k} & =\left(C_{k}+\Delta C_{k}\right) x_{k}+F_{k}^{s} f_{k}^{s}+v_{k},
\end{aligned}
$$

where the state variables are pitch angle $\delta_{z}$, pitch rate $w_{z}$, and normal velocity $\eta_{y}$, the control input $u_{k}$ is the elevator control signal, and $F_{k}^{a}$ and $F_{k}^{s}$ are the matrices distribution of the actuator fault $f_{k}^{a}$ and sensor fault $f_{k}^{s}$. follows:

The presented system equations (73) can be rewritten as

$$
\begin{aligned}
x_{k+1} & =\left(A_{k}+\Delta A_{k}\right) x_{k}+B_{k} u_{k}+F_{k}^{x} f_{k}+w_{k}, \\
y_{k} & =\left(C_{k}+\Delta C_{k}\right) x_{k}+F_{k}^{y} f_{k}+v_{k},
\end{aligned}
$$

where $F_{k}^{x}$ and $F_{k}^{y}$ are the matrices injection of the faults vector in the state and the measurement equations.

$$
\begin{aligned}
& F_{k}^{x}=\left[\begin{array}{ll}
F_{k}^{a} & 0
\end{array}\right], \\
& F_{k}^{y}=\left[\begin{array}{ll}
0 & F_{k}^{s}
\end{array}\right] .
\end{aligned}
$$


The system parameter matrices are

$$
\begin{aligned}
& A_{k} \\
& =\left[\begin{array}{ccc}
0.9944 & -0.1203 & -0.4302 \\
0.0017+\delta_{21}(k) & 0.9902+\delta_{22}(k) & -0.0747 \\
0 & 0.8187 & 0
\end{array}\right], \\
& B_{k}=\left[\begin{array}{c}
0.4252 \\
-0.0082 \\
0.1813
\end{array}\right] \\
& C_{k}=I_{3 \times 3} \text {, } \\
& x_{k}=\left[\begin{array}{lll}
\eta_{y} & w_{z} & \delta_{z}
\end{array}\right]^{T} \text {, } \\
& Q_{k}=\operatorname{diag}\left\{0.1^{2}, 0.1^{2}, 0.01^{2}\right\} \text {, } \\
& R_{k}=0.1^{2} I_{3 \times 3} \text {, }
\end{aligned}
$$

where $\delta_{21}(k)$ and $\delta_{22}(k)$ represent parametric uncertainties in the state matrix satisfying

$$
\begin{aligned}
& \left|\delta_{21}(k)\right| \leq 0.0008 \\
& \left|\delta_{22}(k)\right| \leq 0.05
\end{aligned}
$$

We inject simultaneously two faults in the system:

$$
\left[\begin{array}{c}
f_{k}^{a} \\
f_{k}^{s}
\end{array}\right]=\left[\begin{array}{c}
4 u_{s}(k-40)-4 u_{s}(k-70) \\
-4 u_{s}(k-60)+4 u_{s}(k-80)
\end{array}\right],
$$

where $u_{s}(k)$ is the unit-step function. The first fault $f_{k}^{a}$ occurs in the actuator and the second fault $f_{k}^{s}$ occurs in the sensor for $\delta_{z}$.

The matrices injection of the fault and the unknown disturbances is taken as follows:

$$
\begin{aligned}
& F_{k}^{a}=\left[\begin{array}{c}
0.4252 \\
-0.0082 \\
0.1813
\end{array}\right], \\
& F_{k}^{s}=\left[\begin{array}{l}
0 \\
0 \\
1
\end{array}\right] .
\end{aligned}
$$

In the simulation we set $u_{k}=10, x_{0}=0$, and $P_{0}=0.01^{2} I_{3 \times 3}$.

In Figure 1, we have plotted the actual and the estimated value of the first element and the second element of the fault vector $f_{k}=\left[\begin{array}{ll}f_{k}^{a} & f_{k}^{s}\end{array}\right]^{T}$, respectively, using the RPF and the RPIF. Figure 1 presents the simulation results for the worst case $\left(\delta_{21}(k)=0.0008\right.$ and $\left.\delta_{22}(k)=0.05\right)$. In Figure 2, we have plotted the actual and the estimated value of the state vector $x_{k}=\left[\begin{array}{lll}\eta_{y} & w_{z} & \delta_{z}\end{array}\right]^{T}$ for the two worst cases $\left(\delta_{21}(k)=0.0008\right.$ and $\left.\delta_{22}(k)=0.05\right)$.

According to the simulation results, it can be seen that both the proposed filters RPF and RPIF give a better
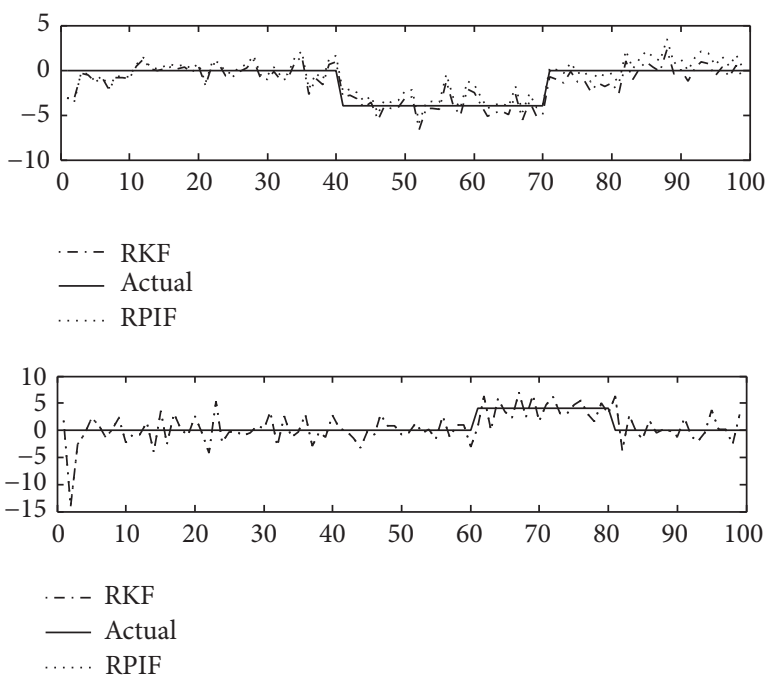

Figure 1: Actual fault $f_{k}$ and estimated fault $\widehat{f}_{k}$.
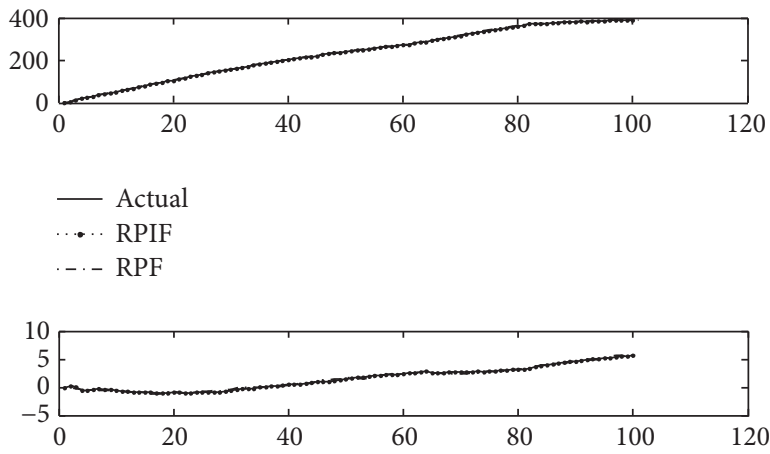

- Actual

- - RPF

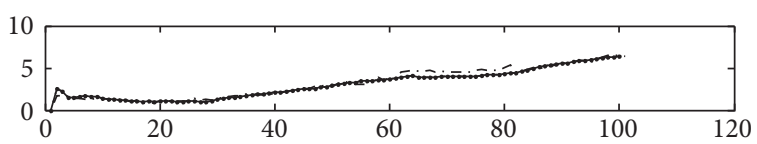

- Actual

... RPIF

$\cdot-\cdot-\mathrm{RPF}$

FIgURE 2: Actual state $x_{k}$ and estimated state $\hat{x}_{k}$.

estimation of the state and the faults. Mainly, we focus on the simultaneous estimation of the actuator and the sensor faults in spite of norm bounded uncertainties in the state and the observation matrix.

Figures 3 and 4 present, respectively, the actual and the estimated value of the first element of the fault vector $f_{k}^{a}$ using the RPF and the RPIF. We can conclude that in the case of time-varying faults the RPIF gives a better estimation results than the RPF. So the integral action is believed to improve robustness against uncertainties and to improve the estimation of time-varying faults. 

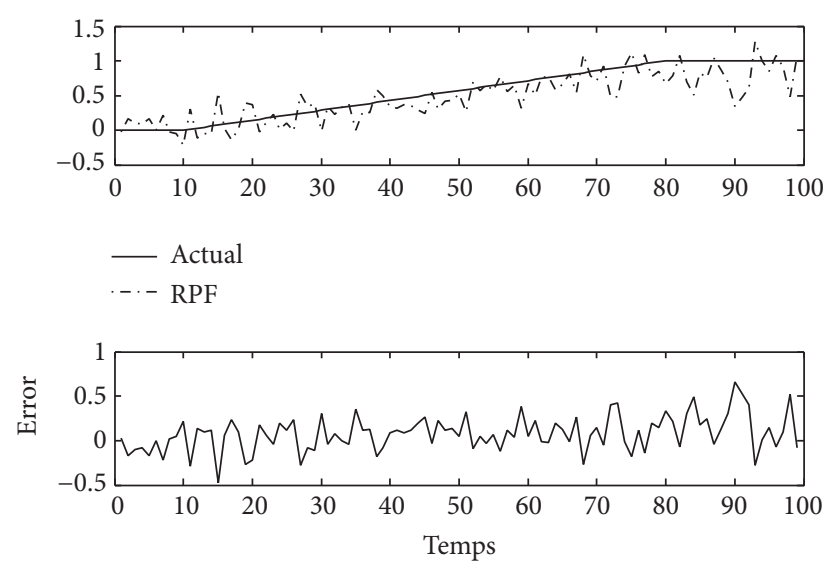

FIGURE 3: Actual fault $f_{k}$ and estimated fault $\widehat{f}_{k}$ (using RPF).
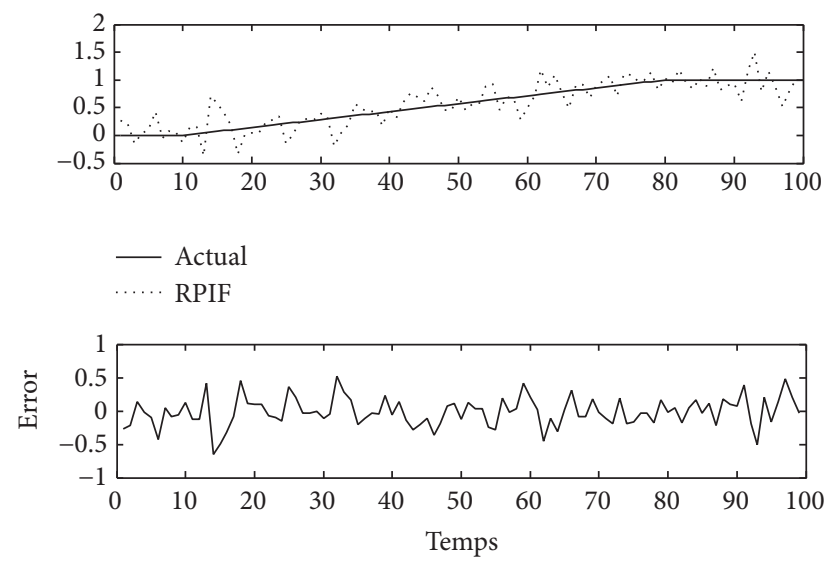

FIGURE 4: Actual fault $f_{k}$ and estimated fault $\widehat{f}_{k}$ (using RPIF).

\section{Conclusion}

In this paper, we have derived a new robust filter for linear time-varying discrete-time systems with unknown faults that affect both the system and the output. By including the unknown fault vector as a part of the augmented system state and using transformation of the original system, a new robust proportional filter (RPF) is developed to solve the robust estimation problem, further, and show how to enforce certain minimum error variance property. We design robust filters that bound the state error covariance matrix for all admissible uncertainties. The robustness criterion used is based on robust square estimation approach. We have extended the developed filter to further propose a new robust proportional integral filter (RPIF) to improve robust estimation of unknown faults and to improve robustness against uncertainties. The design procedure is through the solution of a robust weighted recursive least square problem and it enforces a minimum state error variance property. The advantages of this filter are especially important in the case when the direct feedthrough matrix of the fault has an arbitrary rank and when we do not have any prior information about the fault. An application of the proposed filter has been shown by an illustrative example. The proposed robust filter is able to obtain a robust state and fault estimation in spite of the presence of norm bounded uncertainties. In the next step, we can consider nonlinear systems and the extended Kalman filter can be used to solve the problem of simultaneous fault and state estimation.

\section{Competing Interests}

The authors declare that they have no competing interests.

\section{References}

[1] P. K. Kitanidis, "Unbiased minimum-variance linear state estimation," Automatica, vol. 23, no. 6, pp. 775-778, 1987.

[2] F. Gannouni and F. Ben Hmida, "Robust fault and state-space estimation for linear uncertain systems: an RLS approach," in Proceedings of the 1st International Conference on Electrical Engineering and Software Applications (ICEESA '13), Hammamet, Tunisia, March 2013.

[3] F. Ben Hmida, K. Khémiri, J. Ragot, and M. Gossa, “Unbiased minimum-variance filter for state and fault estimation of linear time-varying systems with unknown disturbances," Mathematical Problems in Engineering, vol. 2010, Article ID 343586, 17 pages, 2010.

[4] F. Ben Hmida, K. Khémiri, J. Ragot, and M. Gossa, "Robust filtering for state and fault estimation of linear stochastic systems with unknown disturbance," Mathematical Problems in Engineering, vol. 2010, Article ID 591639, 24 pages, 2010.

[5] D. Krokavec, A. Filasová, and P. Liščinský, "Unitary approximations in fault detection filter design," Mathematical Problems in Engineering, vol. 2016, Article ID 7249803, 14 pages, 2016.

[6] J. Chen and R. Patton, "Optimal filtering and robust fault diagnosis of stochastic system with unknown disturbances," IEE Proceeding-Control Theory Application, vol. 43, pp. 31-36, 1996.

[7] J. Chen and R. Patton, Robust model-based fault diagnosis for dynamic systems, Kluwer Academic Publishers, 1999.

[8] M. Blanke, M. Kinnaert, J. Lunze, and M. Staroswiecki, Diagnosis and Fault-Tolerant Control, Springer, Berlin, Germany, 2006.

[9] L. Xie, Y. C. Soh, and C. E. de Souza, "Robust Kalman filtering for uncertain discrete-time systems," IEEE Transactions on Automatic Control, vol. 39, no. 6, pp. 1310-1314, 1994.

[10] M. Fu, E. de Souza, and L. Xie, " $H_{\infty}$ estimation for discretetime linear uncertain systems," International Journal of Robust and Nonlinear Control, vol. 2, pp. 87-105, 1991.

[11] X. Zhu, Y. C. Soh, and L. Xie, "Design and analysis of discretetime robust Kalman filters," Automatica, vol. 38, no. 6, pp. 10691077, 2002.

[12] M. Fu, C. E. de Souza, and Z.-Q. Luo, "Finite-horizon robust Kalman filter design," IEEE Transactions on Signal Processing, vol. 49, no. 9, pp. 2103-2112, 2001.

[13] Y. S. Hung and F. Yang, "Robust $H_{\infty}$ filtering with error variance constraints for discrete time-varying systems with uncertainty," Automatica, vol. 39, no. 7, pp. 1185-1194, 2003.

[14] L. Xie, L. Lu, D. Zhang, and H. Zhang, "Improved Robust $\mathrm{H}_{2}$ and $\mathrm{H}_{\mathrm{\infty}}$ filtering for uncertain Discrete-time Systems," Automatica, vol. 40, pp. 873-880, 2004.

[15] Z. Duan, J. Zhang, C. Zhang, and E. Mosca, "Robust $\mathrm{H}_{2}$ and $H_{\infty}$ filtering for uncertain linear systems," Automatica, vol. 42, no. 11, pp. 1919-1926, 2006. 
[16] D. P. Bertsekas and I. B. Rhodes, "Recursive state estimation for a set-membership description of uncertainty," IEEE Transactions on Automatic Control, vol. AC-16, pp. 117-128, 1971.

[17] B. D. O. Anderson and J. B. Moore, Optimal Filtering, PrenticeHall, Upper Saddle River, NJ, USA, 1979.

[18] A. H. Sayed, "A framework for state-space estimation with uncertain models," IEEE Transactions on Automatic Control, vol. 46, no. 7, pp. 998-1013, 2001.

[19] A. Subramanian and A. H. Sayed, "Regularized robust filters for time-varying uncertain discrete-time systems," IEEE Transactions on Automatic Control, vol. 49, no. 6, pp. 970-976, 2004.

[20] O. Y. Bas, B. Shafai, and S. P. Linder, "Design of optimal gains for the proportional integral Kalman filter with application to single particle tracking," in Proceedings of the 38th IEEE Conference on Decision and Control (CDC '99), pp. 4567-4571, Phoenix, Ariz, USA, December 1999.

[21] J. Jung, S. Han, and K. Huh, "Robust proportional-integral Kalman filter design using a convex optimization method," Journal of Mechanical Science and Technology, vol. 22, no. 5, pp. 879-886, 2008.

[22] R. Nikoukhah, "Innovations generation in the presence of unknown inputs: application to robust failure detection," Automatica, vol. 30, no. 12, pp. 1851-1867, 1994.

[23] J. Keller, "Fault isolation filter design for linear stochastic systems with unknown inputs," in Proceedings of the 37th IEEE Conference on Dicision and Control, Tampa, Fla, USA, December 1998.

[24] K. Khémiri, F. Gannouni, F. Ben Hmida, J. Ragot, and M. Gossa, "Robust fault and state estimation for linear discretetime systems with unknown disturbances using PI three-stage Kalman filter," in Proceedings of the International Conference on Communications, Computing and Control Application (CCCA '11), Hammamet, Tunisia, March 2011.

[25] K. Khémiri, F. Ben Hmida, J. Ragot, and M. Gossa, "Novel optimal recursive filter for state and fault estimation of linear stochastic systems with unknown disturbances," International Journal of Applied Mathematics and Computer Science, vol. 21, no. 4, pp. 629-637, 2011.

[26] C. S. Hsieh, "Optimal filtering for systems with unknown inputs via descriptor Kalman filtering," in Proceedings of the 8th International Conference on Control and Automation, pp. 655660, Xiamen, China, 2010.

[27] R. Nikoukhah, A. S. Willsky, and B. C. Levy, "Kalman filtering and Riccati equations for descriptor systems," IEEE Transactions on Automatic Control, vol. 37, no. 9, pp. 1325-1342, 1992.

[28] D. S. Bernstein, Matrix Mathematics: Theory, Facts, and Formulas with Application to Linear Systems Theory, Princeton University Press, Princeton, NJ, USA, 2005. 


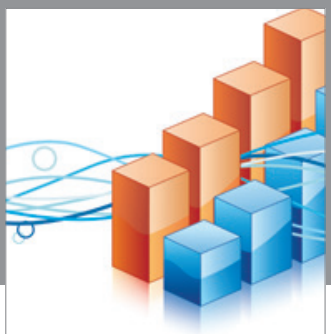

Advances in

Operations Research

vatem alat4

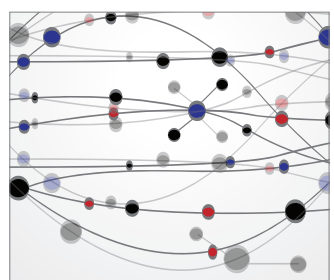

\section{The Scientific} World Journal
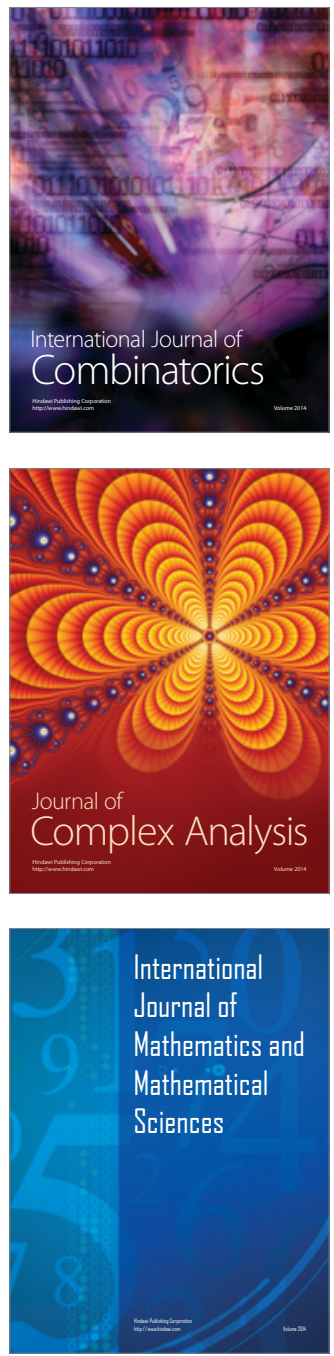
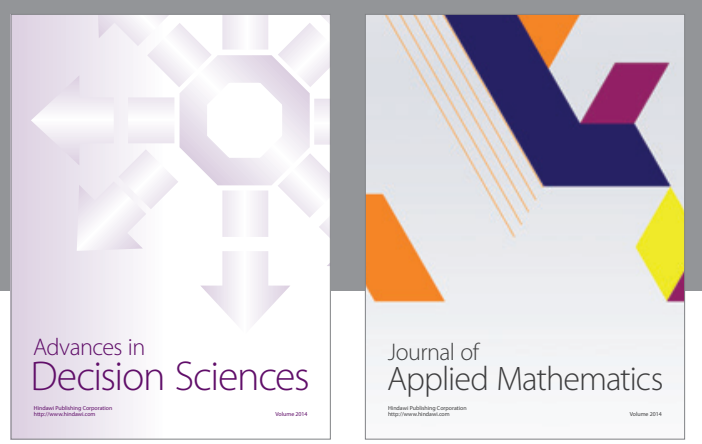

Algebra

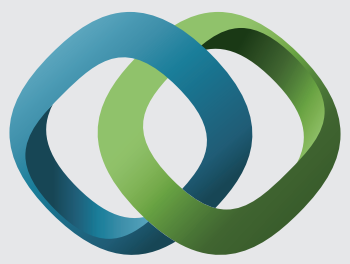

\section{Hindawi}

Submit your manuscripts at

https://www.hindawi.com
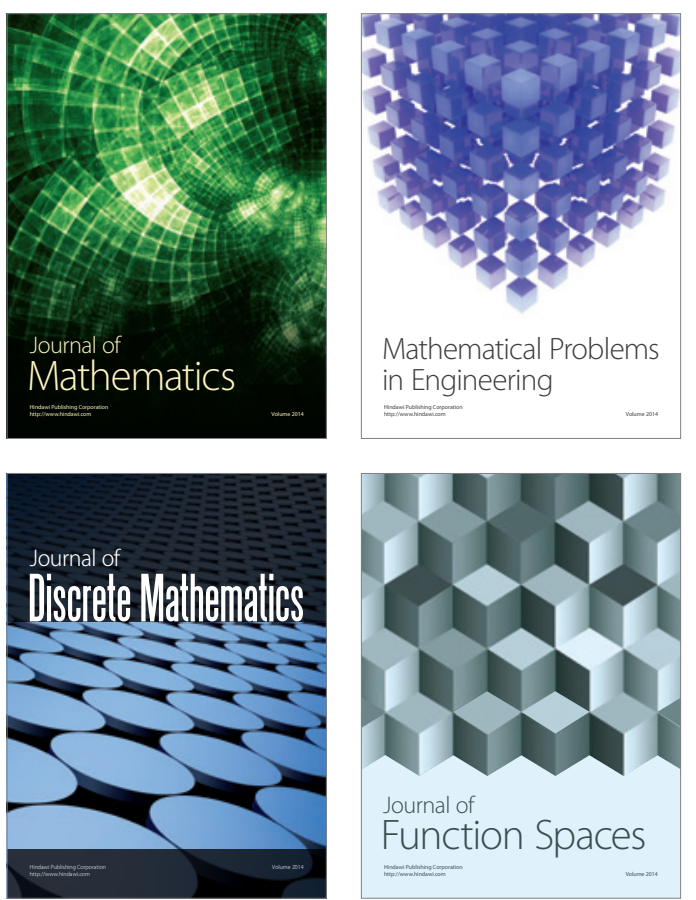

Mathematical Problems in Engineering
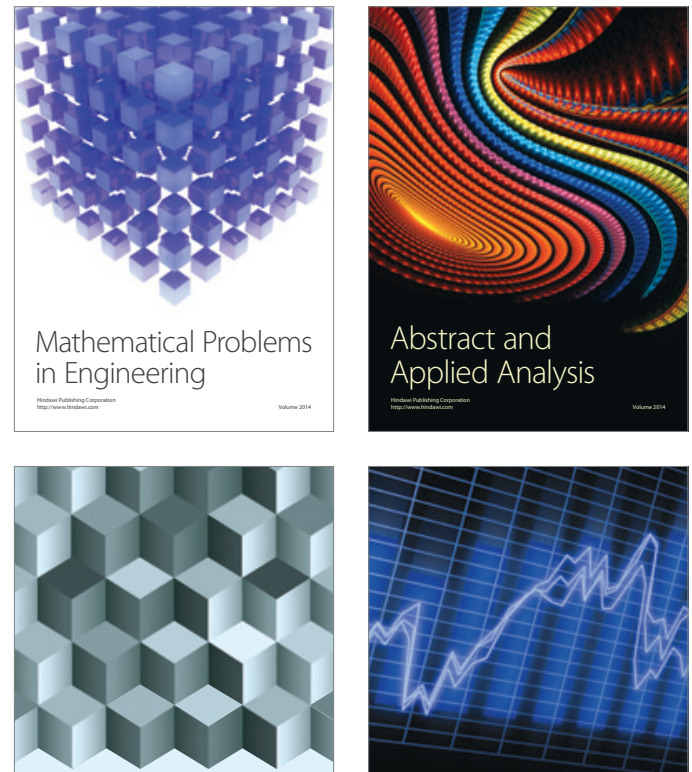

Journal of

Function Spaces

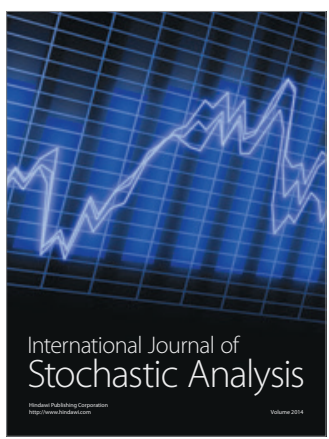

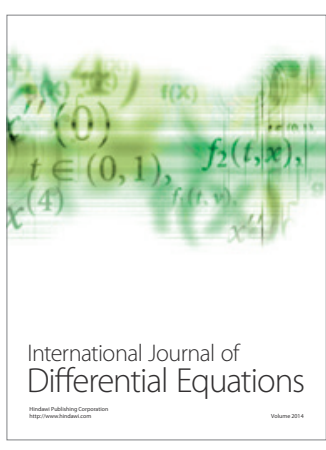
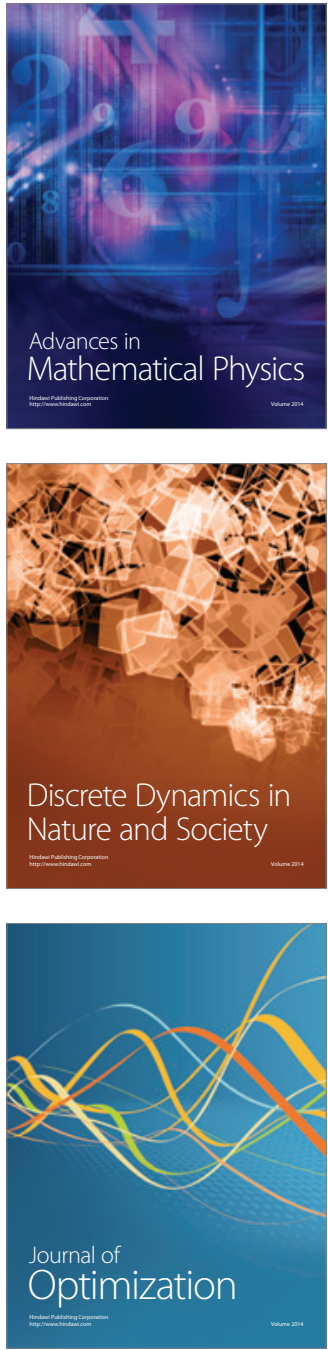